Gut, 1963, 4, 174

\title{
Function of the pylorus and pyloric antrum in gastric emptying
}

\author{
A. K. ARMitage AND A. C. B. DEAN \\ From the Department of Surgery, King's College Hospital Medical School, London
}

EDITORIAL SYNOPSIS The function of the pylorus and the pyloric antrum in the control of gastric emptying has been studied in an isolated rat stomach-duodenum preparation. Pressures in the body and pyloric antrum and flow through the duodenum were recorded using electronic transducers. Vigorous peristalsis and episodic flow were induced by electrical stimulation. The pylorus is normally open until it is transiently closed by an advancing peristaltic wave. High pressures could develop in the antrum when it was separated from the body by another peristaltic wave. Exclusion of the pylorus had very little effect on the pressures developed in body and antrum. The clinical implications of these findings are discussed.

In recent years detailed studies of pressures in the body, antrum and duodenum have been made to investigate the mechanics of gastric emptying. Continuous measurement of the changing flow from the stomach to the duodenum is essential for correlating intragastric pressures with the emptying of gastric contents but this is extremely difficult in patients and conscious animals. By using a preparation in vitro, however, pressure in different parts of the stomach and the flow produced can be studied simultaneously. Experiments in vitro have the additional advantage that the mechanical and pharmacological control can be studied uncomplicated by extrinsic factors. Much of our recent knowledge of the physiology of peristalsis has in fact been obtained from such studies (Bülbring, 1957-58; Bülbring and Crema, 1958).

Recent investigations, such as those of Atkinson, Edwards, Honour and Rowlands (1957) and Edwards (1961), show that the pylorus does not act as an independent sphincter controlling the exit of gastric contents, and the results reported here provide further evidence that the pylorus functions as an integral part of the antrum.

\section{METHOD}

The preparation and the method of pressure recording have been described in detail in a previous paper (Armitage and Dean, 1962). The stomach and duodenum were suspended in oxygenated Krebs' solution as shown in Figure 1. Any desired pressure could be applied to the stomach by varying the height of a Marriotte bottle

'Some of the results reported here were communicated to the Surgical Research Society meeting at Manchester, May, 1962. connected to the body cannula at X. Pressure in the body of the stomach was recorded by an electronic transducer $\left(T_{1}\right)$ attached by an air-filled tube to the second limb of the body cannula at $Y$. Pressure in the antrum was recorded by a transducer $\left(T_{2}\right)$ attached by a saline-filled tube to the antral cannula at $\mathrm{Z}$. Blockage of the cannula was prevented by the slow injection of saline through a three-way tap. Fluid passing from the stomach into the duodenum dropped on to a thin rubber dam tied over a small funnel attached to a third transducer $\left(T_{3}\right)$. Each drop caused a transient rise in pressure recorded as a vertical line and the faster the flow, the closer together

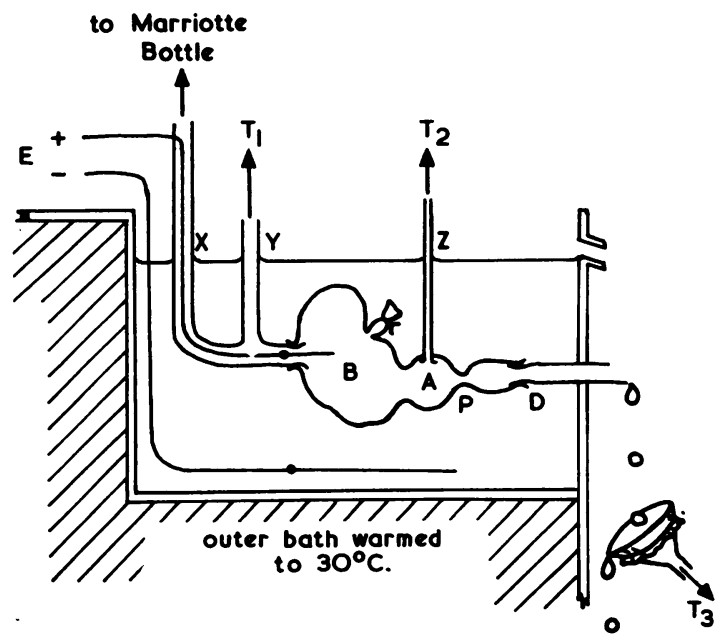

FIG. 1. Diagram of apparatus $T_{1}, T_{2}, T_{3}$, transducers. $E=$ coaxial electrodes, $B=$ body of stomach, $A=$ antrum, $P=$ pylorus, $D=$ duodenum. 
were the lines. When the flow was fast, the base line tended to rise. When flow stopped, the base line fell but this rise and fall was of minor significance and varied from experiment to experiment. It was later found that increasing the sensitivity of the preamplifier and opening the transducer to atmosphere resulted in a steadier base line (Fig. 2). This method of recording flow was designed for these experiments because existing drop recorders were unsuitable for the small but rapidly varying flows encountered.

To induce peristalsis, the preparation was stimulated transmurally (Paton, 1955) using coaxial platinum electrodes $(E)$. Stimuli of frequency $1-5 \mathrm{c} / \mathrm{s}, / \mathrm{msec}$. duration, and 50 volts were used in all experiments. The preparation was stimulated for one-minute periods alternating with one-minute periods without stimulation.

\section{RESULTS}

Spontaneous peristalsis rarely occurred when pres- sure was applied to the stomach. With the pressure at 5 to $10 \mathrm{~cm} . \mathrm{H}_{2} \mathrm{O}$ it was necessary to stimulate the preparation transmurally in order to induce vigorous peristalsis and to maintain it for several hours. If the vagi were isolated and stimulated electrically a similar pattern of pressure and flow was produced. Both hyoscine and hexamethonium blocked the response to either form of stimulation. This means that the response to coaxial stimulation, like that to vagal stimulation, is mediated through parasympathetic nerves. Coaxial stimulation was therefore used as a simple way of producing peristalsis.

Figure 2 shows a typical tracing of the pressures in the body and antrum, and the resulting episodic flow produced by active peristalsis. During each period of stimulation waves of contraction passed along the stomach from the fundus towards the pylorus. At the fundus they were merely shallow ripples but as they

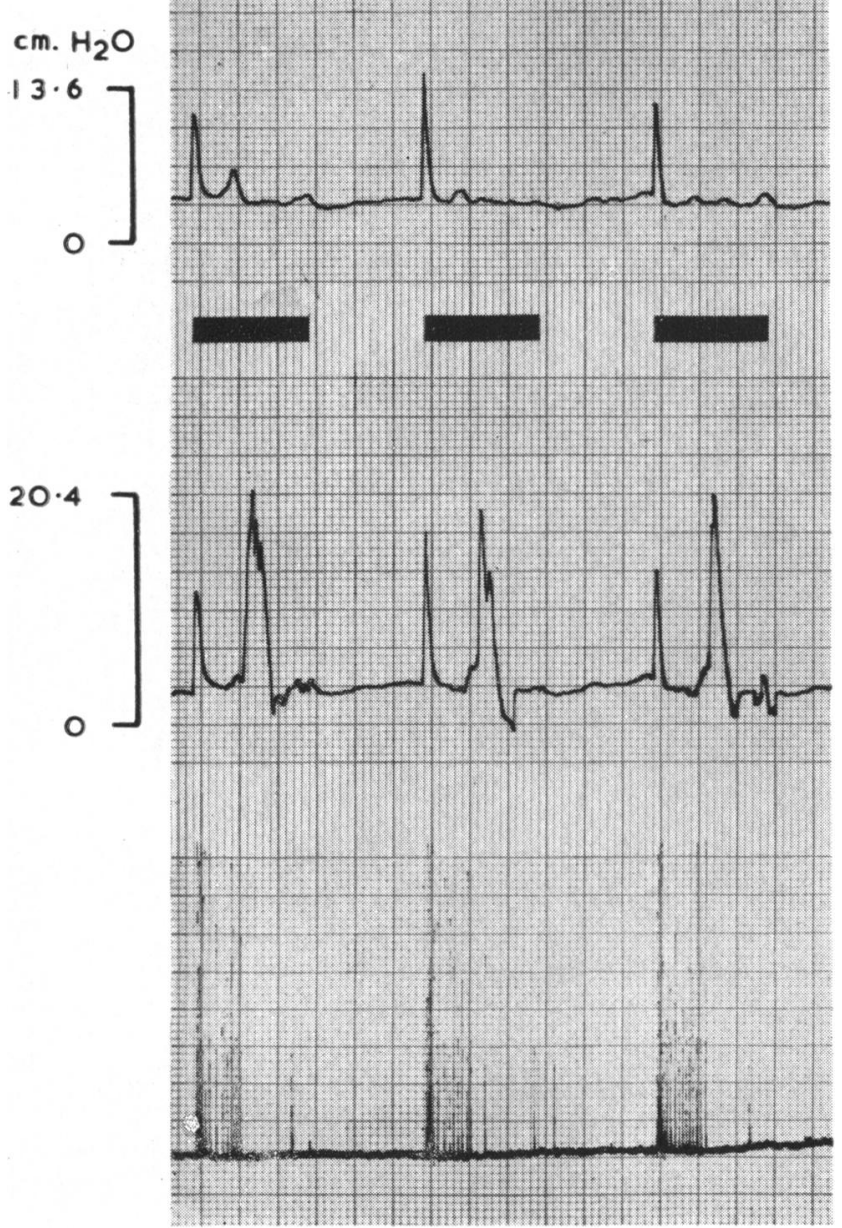

FIG. 2. Tracing of pressure and flow in a rat preparation. Upper tracing, pressure in body of stomach; middle tracing, pressure in antrum;

lower tracing, flow record, each vertical stroke corresponding to one drop.

One-minute periods of stimulation indicated by black bars. Note that peristalsis and flow occurred only during periods of stimulation. 
reached the antrum they became deeper and more vigorous. They divided the stomach into two compartments so that the body was completely separated from the antrum. At this point the pressure in the antrum rose steeply, exceeding that in the body and in some experiments reached $60 \mathrm{~cm} . \mathrm{H}_{2} \mathrm{O}$. Such a pressure difference could not have developed unless the antrum was separated from the body by a peristaltic contraction. The tracing shows that the pylorus opened when stimulation began, and the flow increased as the pressure in both body and antrum rose to about $10 \mathrm{~cm} . \mathrm{H}_{2} \mathrm{O}$. When the pressure began to rise steeply in the antrum the flow into the duodenum stopped abruptly. At this point the pylorus must have been closed, otherwise these high pressures could not have developed. Between periods of stimulation the pressure remained low and there was little flow.

What happens if the pylorus is excluded? This was achieved by advancing the duodenal cannula until its tip had passed through the pylorus, thus preventing it from contracting (Fig. 3). The normal
1

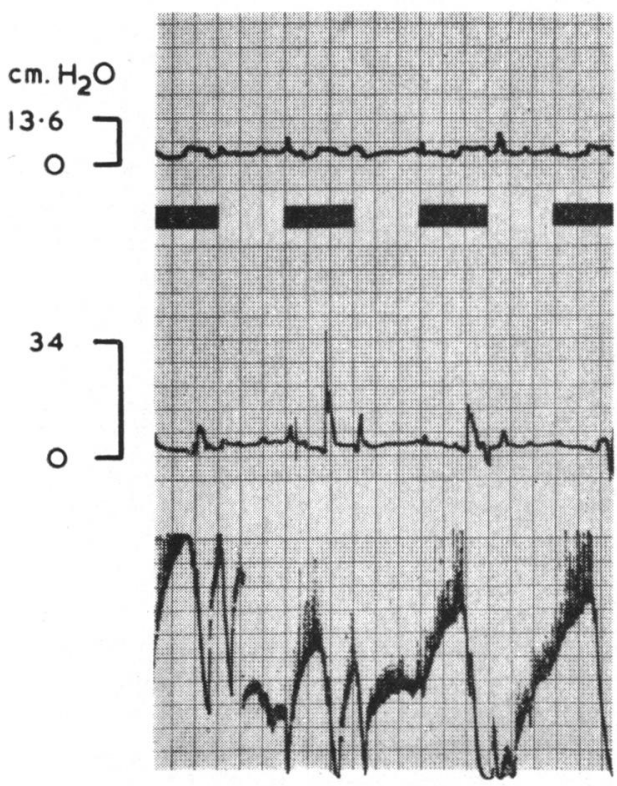

I.

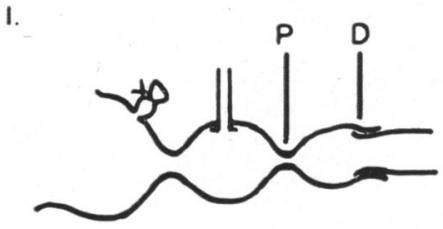

3.

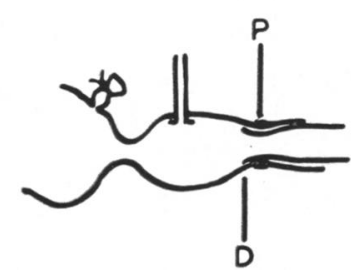

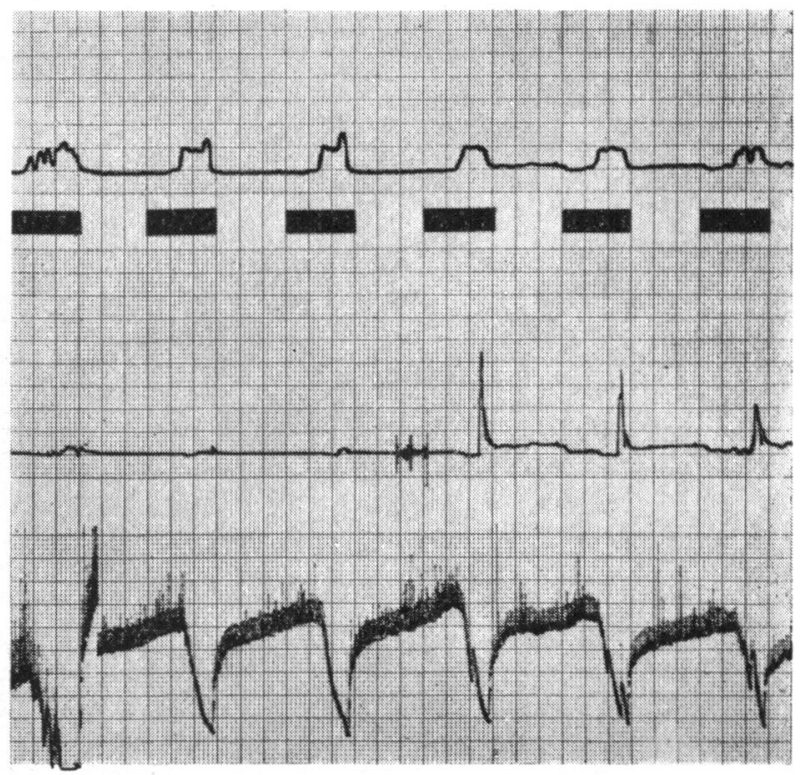

2.

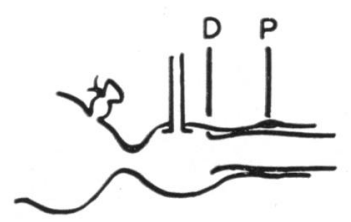

4.

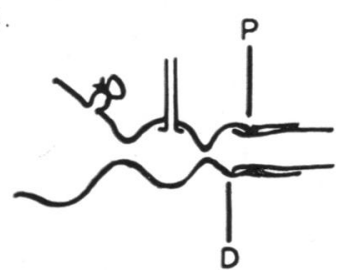

FIG. 3. Rat preparation, record as in Fig. 2: 1, control, with duodenal cannula in usual position; 2, with the duodenal cannula advanced close to the antral pressure recording device, pressures developed in the body (able to form a closed compartment) but not in the antrum; 3 and 4, with the duodenal cannula withdrawn almost to the pylorus, unequal pressures developed in antrum and body (both able to form closed compartments). In 2, 3, and 4 flow in the periods without stimulation was continuous. 
situation is shown on the left, 1 . A peristaltic wave divided the body from the antrum, making it a closed compartment when the pylorus was shut. In 2, the tip of the cannula was pushed well into the antrum. In the tracing it can be seen that pressure waves still occurred in the body but not in the antrum. The body must therefore have been separated from the antrum, which could not develop pressure because it was open to the atmosphere. In 3, the duodenal cannula was withdrawn until the tip only just projected through the pylorus. The tracing shows that the pressure in the antrum now exceeded that in the body because there was now room for a contraction wave to shut off the antrum from the duodenum as shown in 4 . Similar observations were made in each of five experiments.

In order to ascertain whether these results were peculiar to the rat, identical experiments were performed using kittens. Three experiments were done, one of which is illustrated in Figure 4. Transmural stimulation produced coordinated peristalsis and episodic flow (1). In periods without stimulation there was usually little pressure or flow but after pushing the duodenal cannula through the pylorus (2), as in the rat experiments, flow was greatly increased. In the periods of stimulation, waves of contraction still interrupted flow and allowed pressure to develop in the body and the antrum. Although the experiments did not show so striking a pressure differential between body and antrum as in the rat experiments, they did confirm that the distal part of the antrum can act like the pylorus, and that waves of contraction can separate the body from the antrum.
I

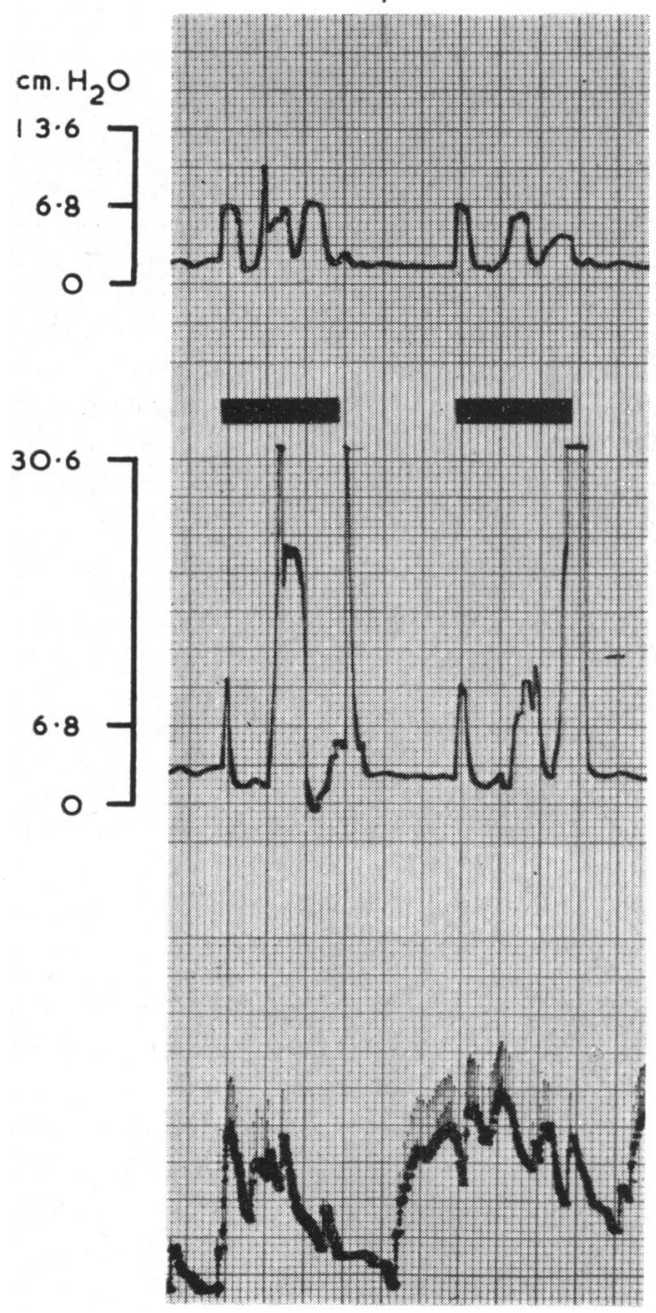

2

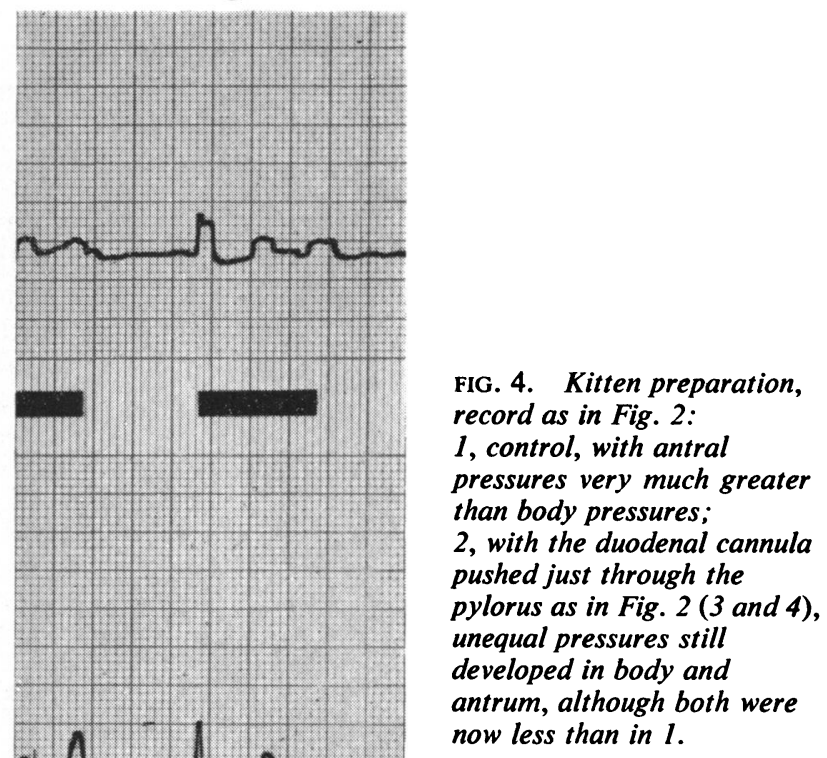




\section{DISCUSSION}

In assessing these findings it should be remembered that in the absence of stimulation the behaviour of the preparation is abnormal. The stomach loses tone, dilates and shows little or no muscular activity. Transmural stimulation, however, simulates normal activity by producing regular peristalsis. Two main observations have been made on the transmurally stimulated stomach. The pylorus is usually open but is transiently closed by advancing peristaltic waves. This confirms the observations of Atkinson et al. (1957). Using miniature balloons, they were unable to demonstrate an area of persistent tone at the gastro-duodenal junction in man. They showed, in agreement with the present results, that high pressures developed in the antrum and pylorus every 20 to 30 seconds, corresponding to the passage of a peristaltic wave. In addition the pressures observed in the preparation in vitro reported here agree closely with those by Smith and Ridgway (1962). In some of our experiments, the pressures in body and antrum differed by a factor of 6 . The possibility of an artificially high pressure being recorded in the antrum as a result of direct pressure from the opposite wall was excluded, since these pressures were observed during the slow continuous injection of saline through the cannula. Atkinson et al. (1957) demonstrated these high pressures in the antrum and pylorus and although they could not measure flows simultaneously, they postulated that the high pressures resulted in powerful propulsion. In our experiments, in which pressure and flow are recorded simultaneously, the highest pressures develop only when there is no flow and when the antrum is shut off from the body and duodenum by successive peristaltic waves. The physiological significance of this is not known. It should, however, be stressed that the present experiments were concerned with the transport of fluid of low viscosity. With normal gastric contents, of a considerably higher viscosity, complete separation may not be necessary for effective propulsion. Our results, showing that high pressures can develop in the antrum while the pressure in the body remains low, indicate that the antrum is separated from the body, and the contraction wave responsible for this division is a feature well known to the gastroscopist. This means that the propulsive activity in the antrum is directed only towards the duodenum and not back into the body as well, as suggested by Edwards (1961).

James (1957) states that solid food remains in the fundus which acts as a 'receptive bag' until it is of such consistency that it can pass into the antrum and be propelled through the pylorus. It may be that this area of constriction between the body and the antrum acts more like a filter than does the pylorus itself.
The role of the pylorus appears to be confined to contracting at the end of each phase of peristalsis. Whether this is to control gastric emptying or to prevent reflux from the duodenum could not be shown in these experiments, but a modification of the technique is being used to investigate this point. We have shown that this closing action is not specific to the pylorus. It can be taken over by the distal part of the antrum when the pylorus is excluded, so that the pattern of pressure and flow is little affected.

\section{CLINICAL IMPLICATIONS}

The fact that higher pressures are developed in the antrum than in the body is clearly of importance in the surgery of the stomach. For example, the rate of emptying of the stomach through a gastroenterostomy will depend on whether the stoma is made in the body or in the antrum. The relatively low pressures in the body may result in slower emptying into the jejunum, while the high pressures developed in the more active antrum will result in more rapid expulsion of the gastric contents.

The effect of pyloroplasty will depend on the length of the incision in the antrum. An extensive incision is likely to distort the powerful circular muscle and result in disturbance of propulsion but this may be compensated by the lowered resistance of the wide outlet.

The overall effects of these operations on gastric emptying are not yet known, but studies such as those reported here suggest that gastric emptying is a dynamic process, and in considering function after gastric surgery the concept of passive drainage is inaccurate and misleading.

We are grateful to Mr. Harold C. Edwards, C.B.E., and Mr. J. G. Murray for their help and encouragement. This work was done during the tenure of personal grants from the Medical Research Council, to whom we wish to express our thanks.

\section{REFERENCES}

Armitage, A. K., and Dean, A. C. B. (1962). A new technique for studying gastric peristalsis in small animals. Wld Med. Electron., 1, 17-19.

Atkinson, M., Edwards, D. A. W., Honour, A. J., and Rowlands, E. N. (1957). Comparison of cardiac and pyloric sphincters. Lancet, 2, 918-922.

Bülbring, E. (1957-58). Physiology and pharmacology of intestinal smooth muscle. Lect. sci. Basis Med., 7, 374-397.

- , and Crema, A. (1958). Observations concerning the action of 5-hydroxytryptamine on the peristaltic reflex. Brit. J. Pharmacol., 13, 444-457.

Edwards, D. A. W. (1961). Physiological concepts of the pylorus. Proc. roy. Soc. Med., 54, 930-933.

James, A. H. (1957). The Physiology of Gastric Digestion. Arnold, London.

Paton, W. D. M. (1955). The response of the guinea-pig ileum to electrical stimulation by coaxial electrodes. J. Physiol. (London), 127, 40-41P.

Smith, A. N., and Ridgway, M. (1962). Application of pressuresensitive telemetering capsules to the study of gastro-intestinal motility. Surgical Research Society meeting, May, 1962. 